\title{
Improving the ADR: Narrow Band, Broad Benefits
}

\author{
Muhammad Aziz ${ }^{1}$. Viveksandeep Thoguluva Chandrasekar²
}

Published online: 12 December 2019

(c) Springer Science+Business Media, LLC, part of Springer Nature 2019

Colonoscopy remains the 'gold standard' for screening colorectal cancer (CRC) with the adenoma detection rate (ADR) being the single most important quality indicator [1]. Corley et al. reported the protective effect of a high ADR, documenting that CRC incidence and mortality were reduced $3 \%$ and $5 \%$, respectively, with every $1 \%$ increase in ADR. They further highlighted the inverse association of a high ADR and interval CRC (HR: 0.52, 95\% CI 0.39-0.69) [2].

In this issue of Digestive Diseases and Sciences, Seo et al. [3] highlight important and ongoing problems faced by endoscopists while performing screening colonoscopies. The authors evaluated the contribution of multiple interventions in improving the colonoscopy outcome metrics for measures such as polyp detection rate (PDR), ADR, advanced adenoma detection rate (AdvADR), cancer detection rate (CaDR), sessile serrated adenoma detection rate (SSADR), and traditional serrated adenoma detection rate (TSADR). The current study emphasizes the importance of a multidisciplinary approach including education, feedback, the use of a split-dose bowel regimen, and the Boston Bowel Preparation Score (BBPS) in improving ADR and other colonoscopy outcome metrics. Each intervention was implemented alone or in combination during different study.

Several techniques have been demonstrated to improve the ADR, especially in low-resource settings including the use of a second observer (fellow, nurse, technician, etc.), employing water-based techniques, the use of addon devices during colonoscopy (cap, endocuff, endorings, G-EYE), enhanced imaging modalities (chromoendoscopy, narrow band imaging, blue laser imaging, and flexible spectral imaging color enhancement), retroflexion in the right

Viveksandeep Thoguluva Chandrasekar viveksandeepvs@gmail.com

1 Department of Internal Medicine, University of Toledo Medical Center, 3000 Arlington Avenue, Toledo, OH 43614, USA

2 Department of Gastroenterology and Hepatology, University of Kansas Medical Center, 3901 Rainbow Blvd, Kansas City, KS 66160, USA colon, and the use of a second forward view [4] and dynamic position change during colonoscopy [5-7].

The variables used in this study by the authors were not novel but have been well established in improving outcomes for colonoscopy. The authors implemented these changes in series during a defined time interval in a stepwise fashion in order to improve the overall ADR in their healthcare system. A considerable improvement in ADR (45.6\% to $48.2 \%$ ), PDR (63.4\% to $72.7 \%$ ), and SSADR (2.1\% to $7.9 \%)$ was noted at the end of the study period. ADR was notably increased with the introduction of and adherence to a splitdose bowel regimen protocol and education of endoscopists regarding adequacy of bowel preparation, consistent with a previous meta-analysis demonstrating reduced ADR with inadequate bowel preparation (OR: 0.53, 95\% CI: 0.46-0.62) [8]. Other aspects noted by Seo et al. that improved ADR included allocating a minimum of 6 min examination time during withdrawal, and education regarding the use of narrow band imaging (although not all endoscopists used it during their exam), using the endoscopic quality improvement program (EQUIP) training for identification of flat polyps, and also endoscopically differentiating neoplastic from nonneoplastic polyps.

More interestingly, the authors demonstrated an impressive increase in SSADR as a possible benefit of education and awareness regarding serrated lesions and the efficacy of narrow band imaging in improving the SSADR, consistent with a recently published meta-analysis that demonstrated the efficacy of narrow band imaging among all the available types of electronic chromoendoscopy in increasing the SSADR: $3.7 \%$ SSADR with NBI and $1.9 \%$ SSADR with high-definition white light imaging (RR: 2.04, 95\% CI 1.18-3.54) [9]. The issue of improving the detection of these lesions still remains, especially in the low-resource setting where expensive modalities such as electronic chromoendoscopy and distal attachments are not available.

The strength of this study is the well-planned methodology with $>10,000$ colonoscopies to help support their results. The results are encouraging with increase in both PDR and ADR at the end of the study period. Even though 
the concept is not novel since similar studies have been conducted in the past, the authors were able to show improved ADR rates even with experienced endoscopists in contrast to previous studies that have not demonstrated improved outcomes with specific planned interventions. It is unclear from this current study as to which intervention could have contributed most to the improved ADR, since there was overlap between different interventions such as the introduction of split-dose bowel preparation and EQUIP training during the same time period. Future quality improvement studies should aim at measuring the improvement in rates for individual outcomes.

Based on the discussion above, gastroenterologists and institutions should implement and target multiple quality measures and interventions in order to improve the overall ADR with consequent reduction in interval CRC. Such institutional quality improvement studies could also help assess which variable contributes to lower ADRs in specific institutions, data that could then be used to target those interventions along with identifying the low ADR endoscopists who could benefit the most from these interventions. There is also the need to explore ways to improve the SSADR, especially in low-resource settings. Future studies targeting the SSADR should be pursued since $20-30 \%$ of colorectal cancers are thought to arise from SSA [10].

Funding No funding was received for the preparation of this manuscript.

\section{Compliance with Ethical Standards}

Conflict of interest The authors declare no conflict of interest.

Human and animal rights No human subjects/animals were involved in this manuscript.

\section{References}

1. Rex DK, Schoenfeld PS, Cohen J, et al. Quality indicators for colonoscopy. Gastrointest Endosc. 2015;81:31-53.

2. Corley DA, Jensen CD, Marks AR, et al. Adenoma detection rate and risk of colorectal cancer and death. $N$ Engl J Med. 2014;370:1298-1306.

3. Seo JY, Jin EH, Bae JH, et al. Multidirectional colonoscopy quality improvement increases adenoma detection rate: results of the Seoul National University Hospital Healthcare System Gangnam Center Colonoscopy Quality Upgrade Project (Gangnam-CUP). Dig Dis Sci. (Epub ahead of print). https://doi.org/10.1007/s1062 0-019-05944-5.

4. Desai M, Bilal M, Hamade N, et al. Increasing adenoma detection rates in the right side of the colon comparing retroflexion with a second forward view: a systematic review. Gastrointest Endosc. 2019;89:453-459.

5. Facciorusso A, Triantafyllou K, Murad MH, et al. Compared abilities of endoscopic techniques to increase colon adenoma detection rates: a network meta-analysis. Clin Gastroenterol Hepatol. 2019;17:2439-2454.e25.

6. Aziz M, Weissman S, Khan Z, et al. Use of 2 observers increases adenoma detection rate during colonoscopy: systematic review and meta-analysis. Clin Gastroenterol Hepatol. 2019. pii: S1542-3565(19)30777-3.

7. Fuccio L, Frazzoni L, Hassan C, et al. Water exchange colonoscopy increases adenoma detection rate: a systematic review with network meta-analysis of randomized controlled studies. Gastrointest Endosc. 2018;88:589-597.e11.

8. Sulz M, Kröger A, Prakash M, et al. Meta-analysis of the effect of bowel preparation on adenoma detection: early adenomas affected stronger than advanced adenomas. PLoS ONE. 2016;11:e0154149.

9. Aziz M, Desai M, Hassan S, et al. Improving serrated adenoma detection rate in the colon by electronic chromoendoscopy and distal attachment: systematic review and meta-analysis. Gastrointest Endosc. 2019;90:721-731.e1.

10. Yamane L, Scapulatempo-Neto C, Resi RM, et al. Serrated pathway in colorectal carcinogenesis. World J Gastroenterol. 2014;20:2634-2640.

Publisher's Note Springer Nature remains neutral with regard to jurisdictional claims in published maps and institutional affiliations. 